\title{
Single-Longitudinal-Mode Diamond Raman Lasers in the Near-Infrared Spectral Region
}

\author{
Oliver Lux ${ }^{1,2}$, Soumya Sarang ${ }^{1}$, Ondrej Kitzler ${ }^{1}$, David J. Spence ${ }^{1}$, Robert J. Williams ${ }^{1}$, Richard P. Mildren ${ }^{1}$ \\ 1. MQ Photonics Research Centre, Department of Physics and Astronomy, Macquarie University, New South Wales 2109 , Australia \\ 2. Deutsches Zentrum für Luft- und Raumfahrt (DLR), Institut für Physik der Atmosphäre, Oberpfaffenhofen 82234, Germany
}

Lasers operating in single-longitudinal-mode (SLM) are of great importance for high-precision measurements in nonlinear optics and spectroscopy as well as for applications in remote sensing, laser cooling and the flourishing field of gravitational wave detection. However, stable SLM in standing-wave inversion lasers is impeded by spatial hole burning which causes mode instability and can only be overcome at the expense of power limitations and/or higher complexity of the laser system, e.g. by means of injection-seeding, ring or microchip laser designs. As an alternative approach, we demonstrate that the nonlinear optical process of stimulated Raman scattering provides a spatial hole burning free gain which enables the generation of SLM output that is intrinsically stable [1]. The underlying mechanism was harnessed for the development of two compact Raman laser configurations which were realized as external standing-wave cavities, without use of any mode-selective elements, and containing only the CVD diamond Raman-active gain medium. Efficient frequency conversion of a tunable $\mathrm{Yb}$ fiber-amplified distributed feedback (DFB) laser emitting around $1064 \mathrm{~nm}$ to the first- and second-order Stokes components produced SLM output in the near-infrared spectral region at powers up to $7 \mathrm{~W}$, while wavelength tuning over a range of $700 \mathrm{GHz}$ was accomplished by varying the temperature of the DFB pump laser, as depicted in Fig. 1a.

The first Stokes diamond Raman laser provided tunable SLM output around $1240 \mathrm{~nm}$ with frequency stability of $80 \mathrm{MHz}$. Without cavity length stabilization, output powers up to $4 \mathrm{~W}$ could be achieved stably for several tens of seconds. Heating in the diamond was found to play an important role in longitudinal mode competition, as it introduces a change in Raman frequency but more importantly alters the optical cavity length through thermal expansion and the thermo-optic effect. In a next step, active stabilization of the cavity length using the HänschCouillaud method [2] was used to maintain SLM operation over longer periods and increase the maximum SLM output power to more than $7 \mathrm{~W}$.

Selective amplification of the second-order Stokes component in a further standing-wave Raman laser generated narrowband output in the eye-safe spectral region around $1486 \mathrm{~nm}$. Here, the implementation of a volume Bragg grating, which provided optical feedback into the Raman cavity, improved the frequency stability to $50 \mathrm{MHz}$ (Fig. 1b). The second Stokes Raman laser was successfully employed for water vapor detection, thus confirming the feasibility of the developed concept for light detection and ranging (LIDAR) applications [3]. Hence, SLM Raman lasers represent a promising alternative to existing OPO/OPA and erbium-based laser sources currently applied for active remote sensing. In general, the results foreshadow a novel approach for greatly extending the power and wavelength range of SLM sources.
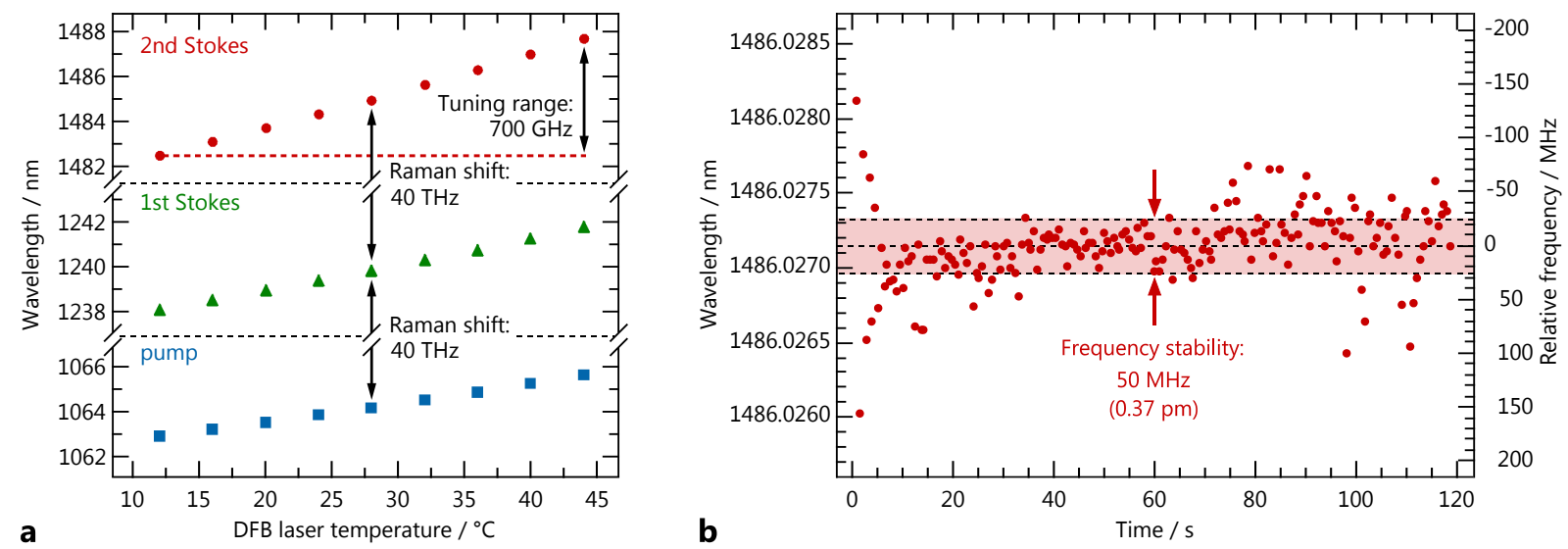

Fig. 1. a Temperature-tuning of the DFB pump laser and resulting output wavelengths of the first and second-order Stokes SLM diamond Raman lasers. b Wavelength stability of the second-order Stokes SLM diamond Raman laser over two minutes.

\section{References}

[1] O. Lux, S. Sarang, O. Kitzler, D. J. Spence, and R. P. Mildren, "Intrinsically stable high-power single longitudinal mode laser using spatial hole burning free gain," Optica 3, 876 (2016).

[2] T. W. Hänsch and B. Couillaud, "Laser frequency stabilization by polarization spectroscopy of a reflecting reference cavity," Opt. Commun. 35, 441 (1980).

[3] O. Lux, S. Sarang, R. J. Williams, A. McKay, and R. P. Mildren, "Single longitudinal mode diamond Raman laser in the eye-safe spectral region for water vapor detection," Opt. Express 24, 27812 (2016). 